\title{
Investigation of Oxygen Diffusion into Contact Lenses Using Electron Spin Resonance Spectroscopy
}

\author{
Emine Aydin, ${ }^{1}$ Evrim E. Özgür, ${ }^{2}$ Turan Özbey, ${ }^{2}$ Murat İrkeç, ${ }^{3}$ Banu Bozkurt, ${ }^{4}$ H. Yılmaz Kaptan ${ }^{2}$ \\ ${ }^{1}$ Turkish Atomic Energy Authority, Ankara Nuclear Research and Training Centre, Besevler, Ankara, 06100, Turkey \\ ${ }^{2}$ Hacettepe University, Department of Physics Engineering, Beytepe, Ankara, 06800, Turkey \\ ${ }^{3}$ Hacettepe University, Department of Ophthalmology, Sihhiye, Ankara, 06100, Turkey \\ ${ }^{4}$ Specialist in Ophthalmology, Private Practice, Turkey
}

Received 18 June 2004; accepted 28 March 2005

DOI 10.1002/app.22536

Published online in Wiley InterScience (www.interscience.wiley.com).

\begin{abstract}
Oxygen permeability is the most important parameter of contact lenses, as lack of oxygen causes corneal edema and threatens the vision of the patient. This study was unique in that it used an electron spin resonance (ESR) technique to determine the oxygen diffusion coefficient $(D)$ of contact lenses. Although there are many methods and techniques for investigating oxygen diffusion into contact lenses, ESR was used for the first time in this study. The ESR technique is based on the scavenging of radicals produced in lenses by oxygen. As a contact lens is not a paramagnetic substance, it cannot give an ESR spectrum. But it does produce an ESR spectrum after $\gamma$ irradiation. When a vacuumirradiated contact lens is exposed to air, the radicals trapped in the lens are transformed into peroxide radicals by the
\end{abstract}

addition of molecular oxygen to the free radicals, and the ESR spectrum begins to change with time. This effect can be used as a tool to measure oxygen uptake in irradiated contact lenses. The oxygen diffusion coefficient of a contact lens was determined from changes in ESR signal intensity varying with time. The diffusion coefficients of oxygen for a contact lens were determined for rapid decay $[(1.5+0.4)$ $\left.\times 10^{-8} \mathrm{~cm}^{2} / \mathrm{s}\right]$ and slow decay $\left[(1.3+0.3) \times 10^{-9} \mathrm{~cm}^{2} / \mathrm{s}\right]$ in this study. These values are in agreement with the $D$ values given in the literature for polymeric materials used for contact lenses. (C) 2006 Wiley Periodicals, Inc. J Appl Polym Sci 100: 2937-2941, 2006

Key words: ESR; hydrogels; diffusion, irradiation

\section{INTRODUCTION}

A simple definition of a contact lens is that it is a small optical system placed directly on the cornea. Contact lenses can be classified into two categories, rigid and soft, the latter of which can be further classified into hydrophobic and hydrophilic. The last category, soft hydrophilic contact lenses, is the most widely used type of contact lens in the world. ${ }^{1}$

Soft contact lenses are made of thermoset polymer hydrogels. The lenses are soft because the polymer is above its glass-transition temperature. ${ }^{1}$

The term soft when applied to contact lens materials in the past carried with it the connotation of being hydrophilic, or water bearing. Because of the high water content of these materials, they are gas permeable. $^{2}$

Oxygen must pass from the air through the tears, around or through the lens, through the tear layer between the lens and cornea, and into the cornea itself. $^{3}$

Correspondence to: H. Y. Kaptan (kaptan@hacettepe. edu.tr).

Journal of Applied Polymer Science, Vol. 100, 2937-2941 (2006) (C) 2006 Wiley Periodicals, Inc.
Two important factors govern the gas transport across a contact lens: The $D k$ value, which indicates the oxygen permeability of the lens. The percentage of water directly affects the gas permeability of a soft contact lens. The thickness, $\ell$, of a lens is the other critical factor. ${ }^{3}$

The soft contact lens does allow oxygen to move through the lens material from the air. From the physiological point of view, the oxygen transmissibility of a soft contact lens material is its most important physicochemical property. ${ }^{2}$

$D k$ values indicate the ability of oxygen to pass through a substance. This oxygen permeability is a characteristic of the plastic itself and not related to the thickness of the material or the actual amount of gas transported in a given contact lens; it may vary with temperature and water content. ${ }^{3}$

The human eye does not receive adequate blood flow to supply enough oxygen to the eye or to remove enough $\mathrm{CO}_{2}$. For this supply the eye depends primarily on exposure to air. If the contact lens does not provide adequate oxygen permeability, the eye suffers serious health risks. ${ }^{4}$

Permeability $(P)$ is defined in a number of ways according to the operating context involved. In the case of oxygen flowing through lenses, $P$ is defined as the product of a dynamic property, the diffusive co- 
efficient $(D)$, and a thermodynamic property $(k)$, representing oxygen solubility in the water-swollen polymer: $P=D k$

Usually, permeability is given in barrers, ${ }^{5-7}$

$$
1 \text { barrer }=10^{-10} \frac{\mathrm{cm}^{3} \mathrm{O}_{2}(S T P) \mathrm{cm}^{2}}{\mathrm{~cm}^{3} \text { of polymers } \mathrm{cm} \mathrm{Hg}}
$$

In fact, $k$ is less important than $D$ because $k$ by itself controls neither the rate of penetration of oxygen nor the steady-state diffusion. However, $k$ is probably the property of a soft contact lens material that is most directly influenced by water.

A pure contact lens is not a paramagnetic substance because it has no paramagnetic centers. For this reason it can not give an ESR spectrum. When contact lenses are irradiated with ionizing radiation, free radicals are generated in them, and an ESR spectrometer detects these radicals. Therefore, after $\gamma$ irradiation, it gives an ESR signal. If a contact lens is irradiated in vacuum and then exposed to air, the radicals trapped in the lens are transformed into peroxide radicals by the addition of molecular oxygen to the free radicals. This effect can be used as a tool to measure the oxygen uptake in irradiated material, and it is a well-known technique. $^{8-11}$

Diffusion can be defined as the transport of a material from one point to another point in the system resulting from random movement of the molecules. The movement of each molecule is independent of the others. The concentration of the diffusive material will eventually be equal in the whole structure when it reaches equilibrium.

The laws of diffusion govern the rate of oxygen movement. ${ }^{2}$

The passage of oxygen through a soft lens material occurs in accordance with Fick's laws of diffusion. ${ }^{3}$

The solution of Fick's second law of diffusion in plane sheet systems gives ${ }^{4,12-16}$

$$
\begin{aligned}
\frac{M_{t}}{M_{\infty}}=1-\frac{8}{\pi^{2}} \sum_{n=0}^{\infty} \frac{1}{(2 n+1)^{2}} & \\
& \quad \times \exp \left[-D(2 n+1)^{2} \pi^{2} t / 4 \ell^{2}\right]
\end{aligned}
$$

where $\ell$ is the thickness of the sheet, $D$ is the diffusion coefficient, and $M_{t}$ and $M_{\infty}$ represent the amounts of the diffusant entering the sheets at times $t$ and infinity, respectively.

Using the present technique, it was possible to obtain a decreased ESR signal intensity, $R$, of the radicals with time. It was therefore necessary to find a direct relation between the measured quantity (signal intensity) and the ratio $M_{t} / M_{\infty}$. We related the $M$ values to radical concentration in eq. $(2)^{3}$

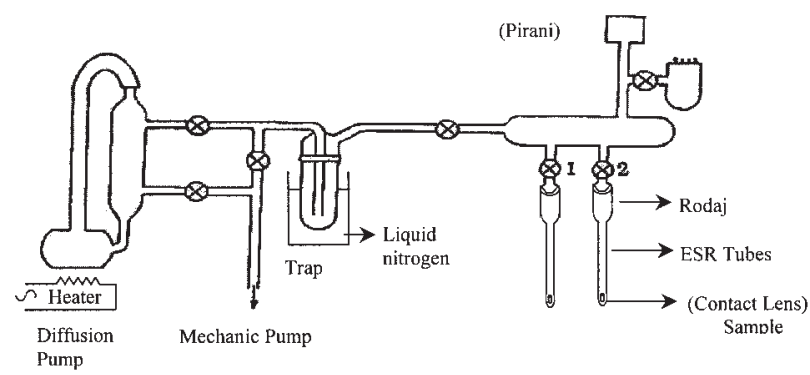

Figure 1 Vacuum system.

$$
\frac{M_{t}}{M_{\infty}}=1-\frac{R_{t}}{R_{0}}
$$

where $R_{0}$ and $R_{t}$ are the primary radical concentrations at $t=0$ and at time $t$, on exposure to air, respectively.

Substitution of eq. (2) into eq. (1) gives

$$
\frac{R_{t}}{R_{0}}=\frac{8}{\pi^{2}} \sum_{n=0}^{\infty} \frac{1}{(2 n+1)^{2}} \cdot \exp \left[-D(2 n+1)^{2} \pi^{2} t / 4 \ell^{2}\right]
$$

We already know that in the expansion of the exponential term under the summation sign in eq. (3) the contribution of higher values of $n$ can be neglected. By combining the $R_{0}$ value with $8 / \pi^{2}$ and considering the $n=0$ case, eq. (3) can be rewritten as

$$
R_{t}=R_{0} \exp \left(-D \pi^{2} t / 4 \ell^{2}\right)
$$

The above equation formed the theoretical basis for determining $D$ in this study. By inspecting the relative change in radical concentration with time, that is, $R_{t} / R_{0}$, of $\gamma$-irradiated contact lenses with a known thickness, $\ell$, it was possible to fit the above equation to the experimental data and determine $D$, the diffusion coefficient of oxygen. The same quantity also could be determined from the slope of the $\ln \left(R_{t} / R_{0}\right)$ versus time graph.

\section{EXPERIMENTAL}

The most commonly used method of measuring the permeability of oxygen of hydrogel contact lenses was first introduced by Fatt and then officially adopted by the International Standard Organization (ISO). ${ }^{5-7,17}$

Contact lens samples with an average thickness of $0.07 \mathrm{~mm}$ were used. These samples were placed in Pyrex ESR tubes and connected to a vacuum line of $10^{-3}$ torr for $1 \mathrm{~h}$ and after that were flame-sealed (Fig. $1)$.

The samples kept in a vacuum were irradiated with a gamma cell PX- $\gamma$-30 Issledovatelj-type ${ }^{60}$ Co gamma source at room temperature at a dose rate of 2.05 


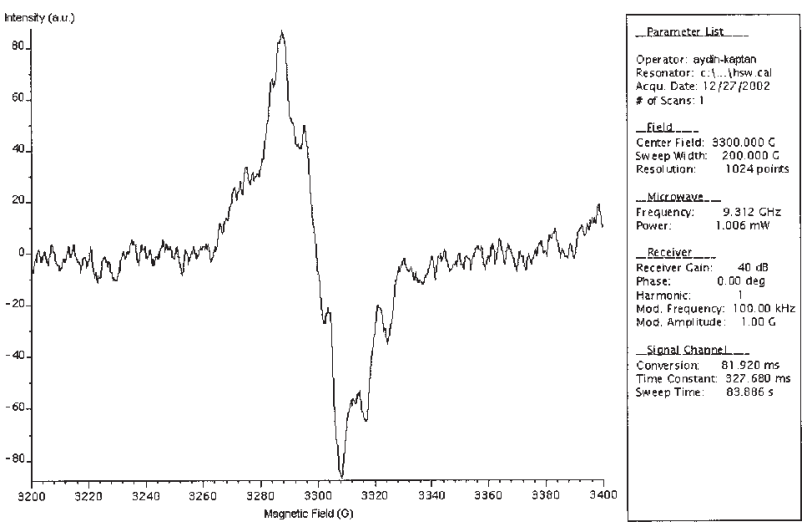

Figure 2 One of the ESR spectra of an irradiated contact lens in vacuum.

$\mathrm{kGy} / \mathrm{h}$. The total dose given to contact lens samples was 2 kGy. This dose was determined from preliminary testing in which samples were exposed to varied doses ranging from 0.5 to $60 \mathrm{kGy}(0.5,1.0,2.0,10,20$, 30, 40, and $60 \mathrm{kGy}$ ), recording the relevant decay curves of each. Analysis of the test results showed that 2 kGy was the optimum dose for the PMMA-based contact lenses because high doses cause microstructural changes and low doses have rapid decay of signal output, thus not allowing sufficient time for data recording.

The paramagnetic centers produced in the ESR tubes were removed by carefully heating the inverted ends of the tubes. These heated ends of the sample tubes were later placed in the cavity of the ESR spectrometer, and the first ESR spectra were taken at time $t=0$. After the tube was opened to the air $(t>0)$, ESR spectra were recorded with time until the following lines were damping completely.

\section{ESR measurements}

The ESR spectra were recorded by a Bruker EMX-131 $X$-band spectrometer with a modulation frequency of $100 \mathrm{kHz}$. Microwave power and modulation amplitude were kept at $1 \mathrm{~mW}$ and $1 \mathrm{G}$, respectively, during the experiments, All ESR spectra were recorded at room temperature.

\section{RESULTS AND DISCUSSION}

An original ESR spectrum of a vacuumed contact lens irradiated at a 2-kGy dose rate is shown in Figure 2. Theoretical study of the source radical of this ESR spectrum is detailed at the end of this section.

Figure 3 shows the original and decayed ESR signals of an irradiated contact lens. The first ESR signal shown is the first spectrum, which was taken in a vacuum (before the tube had been exposed to air) at

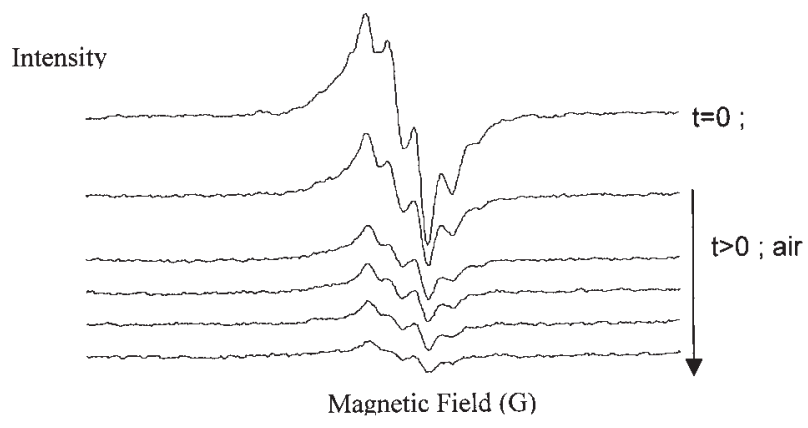

Figure 3 ESR spectrum of irradiated lens in vacuum $(t=0)$ and change in the spectrum after exposure of the tube to air $(t>0)$.

time $t=0$. Because of the vacuum environment, no oxygen molecules were left in the ESR tube. Therefore, this spectrum came from radicals that were produced in the contact lens by irradiation. The other spectra were taken after the tube was opened to the air $(t>0)$. These ESR spectra were recorded with time until the intensities of the curves were damped completely.

Four lines in this spectrum were selected, and decremental changes in their intensity with time were analyzed. Figure 4 shows these curves recorded after air was allowed into the tube. The intensity of each curve is marked as $\mathrm{A}, \mathrm{B}+, \mathrm{B}-$, or $\mathrm{C}$, as shown in Figure 4.

Peak-to-peak intensity of a line was used as the radical concentration, $R_{t}$, because the line-width $\Delta H_{P P}$ remained constant during the experiment. Changes in the intensity or radical concentration of a contact lens with time for those four lines are shown in Figure 5.

It can be seen from Figure 5 that the intensities of all lines decreased with time. It also can be clearly seen from Figure 5 that the decay rates of radicals decreased with oxygen permeation into the lens with increasing time. This was an expected behavior because the radicals produced by irradiation $\left(R^{\circ}\right.$ were transformed into peroxy radicals $\left(\mathrm{RO}_{2}{ }^{\circ}\right.$ by the addition of molecular $\left(\mathrm{O}_{2}\right)$ oxygen to the free radicals:

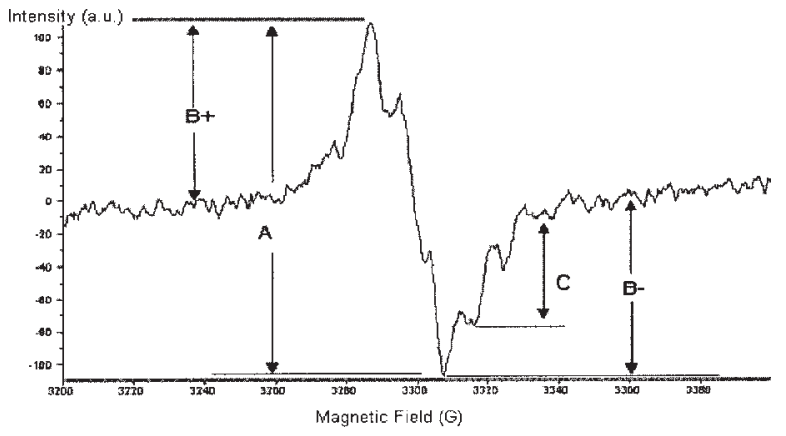

Figure 4 Peak-to-peak intensities of ESR lines were followed during the experiment. 


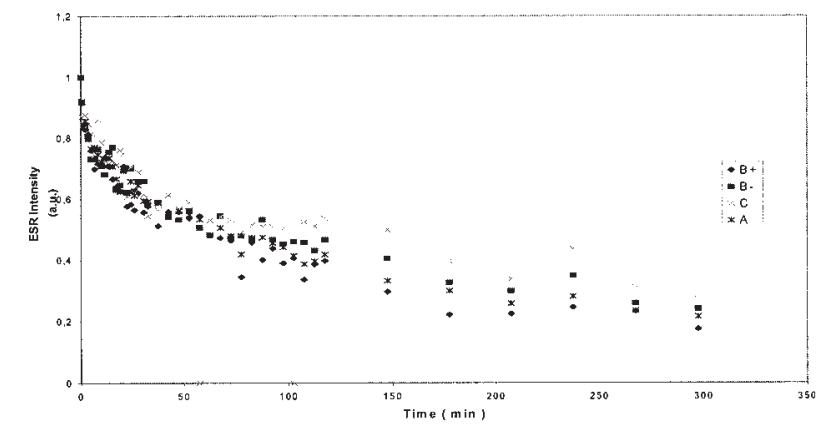

Figure 5 Change in the radical concentration (ESR signal intensity) with time for the four lines of ESR spectra shown in Figure 4

$$
\mathrm{R}^{\cdot}+\mathrm{O}_{2} \rightarrow \mathrm{RO}_{2}^{\cdot}
$$

As a consequence of this reaction, the ESR signal arising from $\mathrm{R}^{*}$ started to lose its intensity and changed its shape, and furthermore it was replaced by a peroxy signal. These effects can be use as a tool to measure oxygen uptake in irradiated material.

By following the relative change in radical concentration with time, that is, $R_{t} / R_{0}$ of $\gamma$-irradiated contact lenses of known thickness, $\ell$, it was possible to plot an $\ln \left(R_{t} / R_{0}\right)$ versus $t$ graph for all lines $(\mathrm{A}, \mathrm{B}+, \mathrm{B}-, \mathrm{C})$ in the ESR spectrum, as shown in Figure 6.

Equation (4) was the theoretical basis for determining $D$ in this study. By using this equation and the slope of the lines in the $\ln \left(R_{t} / R_{0}\right)$ versus time graph, it was possible to determine the diffusion coefficient $(D)$ of the contact lenses.

For each decay curve labeled as $\mathrm{A}, \mathrm{B}+, \mathrm{B}-$, and $\mathrm{C}$ (shown in Fig. 5), an $\ln \left(R_{t} / R_{0}\right) \alpha$ time graph was plotted and a $D$ value determined. These calculated $D$ values are listed in Table I.

As can be seen from Figure 6, there were two linear regions, rapid- and slow-decay regions, which corre-

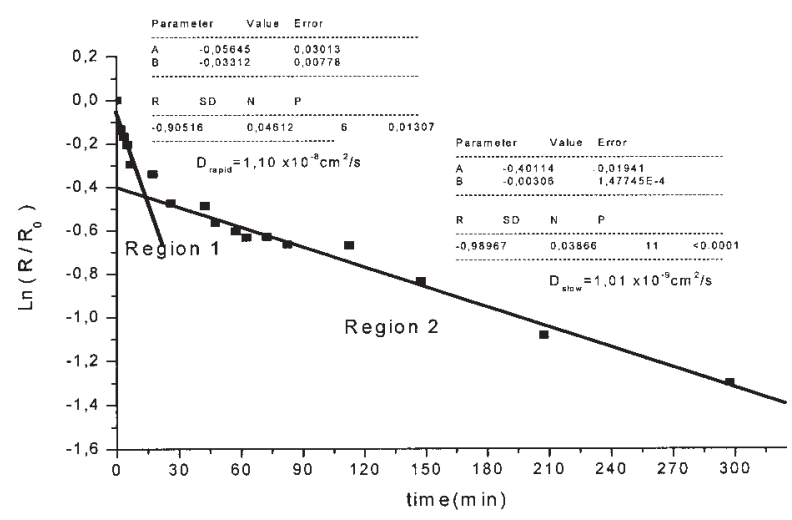

Figure $6 \mathrm{~A} \ln \left(R_{t} / R_{0}\right) \alpha$ time graph and some results of fit for line $C$ in our ESR spectrum.
TABLE I

$D$ values Determined by Following the Lines A, B+, B-, C in ESR Spectrum

\begin{tabular}{lcc}
\hline & \multicolumn{2}{c}{$D\left(\mathrm{~cm}^{2} / \mathrm{s}\right)$} \\
\cline { 2 - 3 } Line & Region 1 & Region 2 \\
\hline A & $1.26 \times 10^{-8}$ & $1.38 \times 10^{-9}$ \\
B + & $1.56 \times 10^{-8}$ & $1.61 \times 10^{-9}$ \\
B- & $1.82 \times 10^{-8}$ & $1.15 \times 10^{-9}$ \\
C & $1.10 \times 10^{-8}$ & $1.01 \times 10^{-9}$ \\
\hline
\end{tabular}

sponded to short- and long-term diffusion, respectively.

The first region, that is, the first $10-15 \mathrm{~min}$ of oxygen, diffused into the system easily because there was no resistance to the oxygen molecules during this time (see Table I). The $D$ values of all the lines were nearly identical $(1.5 \pm 0.4) \times 10^{-8} \mathrm{~cm}^{2} / \mathrm{s}$.

The second region was between 15 and $300 \mathrm{~min}$. And the $D$ values of all the lines were nearly the same $(1.3 \pm 0.3) \times 10^{-9} \mathrm{~cm}^{2} / \mathrm{s}$ and less than those in the first region. This may have been because the oxygen molecules that entered behaved as a barrier, hindering penetration of new oxygen molecules farther into the center of the lens, making the diffusion of oxygen more and more difficult in the deeper parts of the lens.

We think that the diffusion coefficient of the second region $(1.3 \pm 0.3) \times 10^{-9} \mathrm{~cm}^{2} / \mathrm{s}$ was the real diffusion coefficient of the contact lenses used in the present study.

\section{Computer simulations}

To determine the source of the decay curves detected from the ESR spectrum, different microwave power values also were examined. The results showed that the typical ESR spectrum remained similar to the one shown in Figure 2 for each power input. The slopes of the two regions also were observed to be similar for all four decay curves when $\ln \left(R_{t} / R_{0}\right)$ was plotted against time. As a result, it was deduced that the source of the radicals was the same for all decay curves in the ESR spectra.

Experimental data were simulated in order to obtain the theoretical spectrum using a special computer program, SimFonia. The spectroscopic parameters $(g$ value, line width, hyperfine splitting constant, and relative spectral intensity of component radicals) were obtained from the observed spectra.

It has been suggested that the $\mathrm{C}-\mathrm{C}$ bond in the main chain is broken by irradiation, and unpaired electrons occur. These unpaired electrons interact with the three, nearly equivalent protons and cause hyperfine splitting. The best parameters for these hyperfine coupling constants and $g$ values are $A_{x x}=A_{y y}=8.8 \mathrm{G}$, $A_{z z}=8.0 \mathrm{G}$ and $g_{x x}=g_{y y}=2.02050, g_{z z}=2.0149$. 


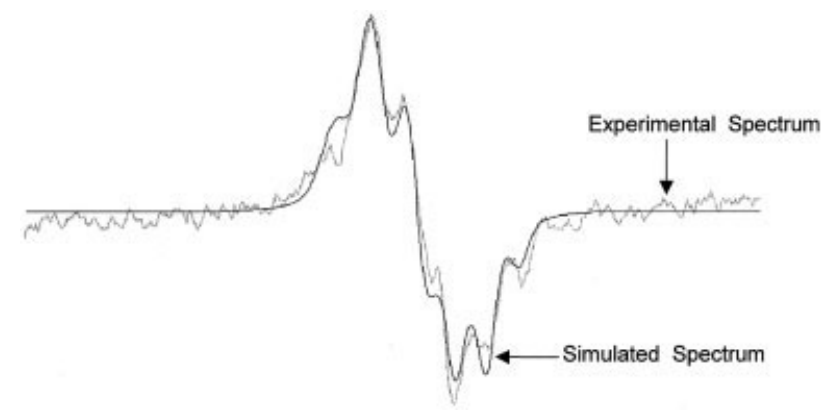

Figure 7 Experimental and simulated spectra of a $2 \mathrm{kGy}-$ irradiated contact lens sample.

The experimental and theoretical (simulated) spectra are shown in Figure 7, in which it can be clearly seen that the two spectra are in good agreement with each other.

Finally, it can be concluded that the present study, believed to be the first to determine oxygen diffusion into soft contact lenses using the ESR technique, was successful. Detailed interdisciplinary studies of oxygen and liquid diffusion into used and unused soft contact lenses currently are being conducted using ESR as a function of time.

\section{References}

1. Bergin, J. Introduction to Polymers; State University of New York at Buffalo: Buffalo, NY, 2000.
2. Fatt, I. Soft Contact Lenses: Clinical and Applied Technology; Ruben, M., Ed.; Wiley: New York, 1978; p 83-110.

3. Phillips, A. J.; Stone, J. Contact Lenses. A Textbook for Practitioner and Student; Butterworths: London, 1989; p 2937.

4. Thimmegowda, M. C.; Sathyanarayana, P. M.; Shariff, G.; Ashalatha, M. B.; Ramani, R.; Ranganathaiah, C. Phys Stat Sol A 2002, 2, 257.

5. Manetti, C.; Casciani, L.; Pescosolido, N. Polymer 2002, 43, 87.

6. Compañ, V.; López, M. L.; Andrio, A.; López-Alemany, A.; Refojo, M. F. J Appl Polym Sci 1999, 72, 321.

7. Compañ, V.; Andrio, A.; López-Alemany, A.; Riande, E.; Refojo, M. F. Biomaterials 2002, 23, 2767.

8. Eken, M.; Turhan, S..; Kaptan, H. Y.; Güven, O. Radiat Phys Chem 1995, 46, 809.

9. Kaptan, H. Y.; Pekcan, Ö.; Güven, O. J Appl Polym Sci 1992, 44, 1595.

10. Kaptan, H. Y.; Pekcan, Ö.; Arca, E.; Güven, O. J Appl Polym Sci 1989, 37, 2577.

11. Kaptan, H. Y.; Güven, O. J Appl Polym Sci 1997, 64, 1291.

12. Crank, J.; Park, G. S. Diffusion in Polymers; Academic Press: London, 1968.

13. Crank, J. The Mathematics of Diffusion; Oxford University Press: London, 1970.

14. Kaptan, H. Y. Ph.D. Thesis, Hacettepe University, Ankara, Turkey, 1987.

15. Kaptan, H. Y.; Pekcan, Ö.; Arca, E.; Güven, O. J Appl Polym Sci 1989, 37, 2577.

16. Poulsen, L.; Ogilby, P. R. J Phys Chem A 2000, 104, 2573.17. Fatt, I.; St. Helen, R. Am J Optom 1971, 48, 545. 